\title{
Unified Field Theory
}

\author{
Parashu Ram Poudel \\ Department of Physics, Prithvi Narayan Campus, Pokhara \\ parashupoudel@hotmail.com
}

\begin{abstract}
Unified field theory is the long-sought means of tying together all known phenomena to explain the nature and behaviour of all matter and energy in existence. The quest for unification has been the perennial theme of modern physics. The belief that all physical phenomena can be reduced to simple and explained by a smaller number of laws is the central tenet of physics. Such a theory could potentially unlock all the secrets of nature and make a myriad of wonders possible, including such benefits as time travel and an inexhaustible source of clean energy, among many others. This paper aims to explain unified theory and its development towards the unification of four interactions in brief.
\end{abstract}

Keywords: graviton, gluons, grand unified theory, theory of everything, super gravity

\section{INTRODUCTION}

Unified field theory is the physical theory that combines the treatment of two or more types of fields in order to deduce previously unrecognized interrelationships, especially such a theory unifying the theories of nuclear, electromagnetic, and gravitational forces. In physics, a field refers to an area under the influence of some force, such as gravity or electricity, for example. A unified field theory would reconcile seemingly incompatible aspects of various field theories to create a single comprehensive set of equations. It is an attempt to describe all fundamental forces and the relationships between elementary particles in terms of a single theoretical framework. The term 'Unified Field Theory' was coined by Einstein. Einstein spent the latter part of his life searching for such a unified field theory, but was unsuccessful.

Physicists believe that laws of classical mechanics are a good approximation to the quantum mechanical laws that govern the interaction of atoms, that the structure of atoms can be explained by the laws of interaction of nuclei and electrons, that the structure of nuclei can be explain by the theory of quarks and gluons, and finally that these are consequences of an even more comprehensive unified theory to be developed.

Each stage in this development has required exploring natural phenomena at shorter and shorter distance, explaining complex macroscopic constituents in terms of simple microscopic constituents. Ordinary matter is made of atoms; atoms are made of nuclei, nuclei made of quarks. At each stage, the laws of physics are more unified explaining a larger class of phenomena and reducing to law of previous in appropriate circumstances. Typically, the more unified theories exhibit more symmetry, are more predictive and less arbitrary.

\section{FUNDAMENTAL FORCES}

According to the current knowledge of physics, forces are not transmitted directly between objects, but instead are described by intermediary entities called fields. All four of the known fundamental forces are mediated by fields, which in the Standard Model of particle physics result from exchange of gauge bosons. Specifically the four interactions to be unified are: Gravitation, Electromagnetism, Weak Interaction and Strong Interaction. They are described briefly as follows:

\subsection{Gravitational Force}

Gravitational force is a purely attractive force which reaches through even the "empty" void of space to draw two masses toward each other. It keeps the planets in orbit around the sun and the moon in orbit around the Earth.

Gravitation is described under the theory of general relativity, which defines it as the curvature of space time around an object of mass. This curvature, in turn, creates a situation where the path of least energy is toward the other object of mass. This interaction acts on all particles. The postulated exchange particle has been named the graviton 


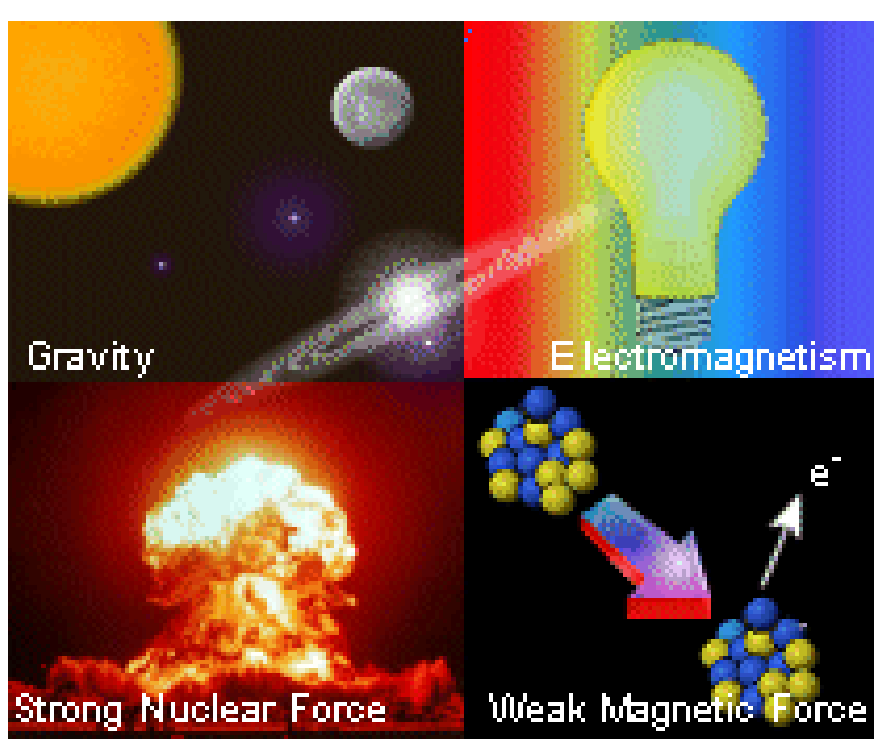

Fig 1: Illustration of the realm of four types of interaction.

\subsection{Electromagnetism}

Electromagnetism is the interaction of particles with an electrical charge. Charged particles at rest interact through electrostatic forces, while in motion they interact through both electrical and magnetic forces. The photon is the exchange particle for this force. For a long time, the electric and magnetic forces were considered to be different forces, but they were finally unified by James Clerk Maxwell in 1864, under Maxwell's equations. In the 1940s, quantum electrodynamics consolidated electromagnetism with quantum physics. Electromagnetism is perhaps the most obviously prevalent force in our world, as it can affect things at a reasonable distance and with a fair amount of force.

\subsection{Weak Force or Interaction}

The weak interaction is a very powerful force that acts on the scale of the atomic nucleus. This repulsive short-range interaction is responsible for some forms of radioactivity, that acts on electrons, neutrinos, and quarks. It causes phenomena such as beta decay. It has been consolidated with electromagnetism as a single interaction called the "electroweak interaction." The weak interaction is mediated by the $\mathrm{W}$ boson (there are actually two types, the $\mathrm{W}^{+}$and $\mathrm{W}^{-}$bosons) and also by the $\mathrm{Z}$ boson.

\subsection{Strong Force or Interaction}

The strong interaction is responsible for holding quarks together to form neutrons and protons, and holding neutrons and protons together to form nuclei. In the helium atom, for example, it is strong enough to bind two protons together despite the fact that their positive electrical charges cause them to repulse each other.

In essence, the strong interaction allows particles called gluons to bind quarks together to create the nucleons in the first place. Gluons can also interact with other gluons, which give the strong interaction a theoretically infinite distance; although it's major manifestations are all at the subatomic level.

Modern unified field theory attempts to bring these four interactions together into a single framework as shown in figure.

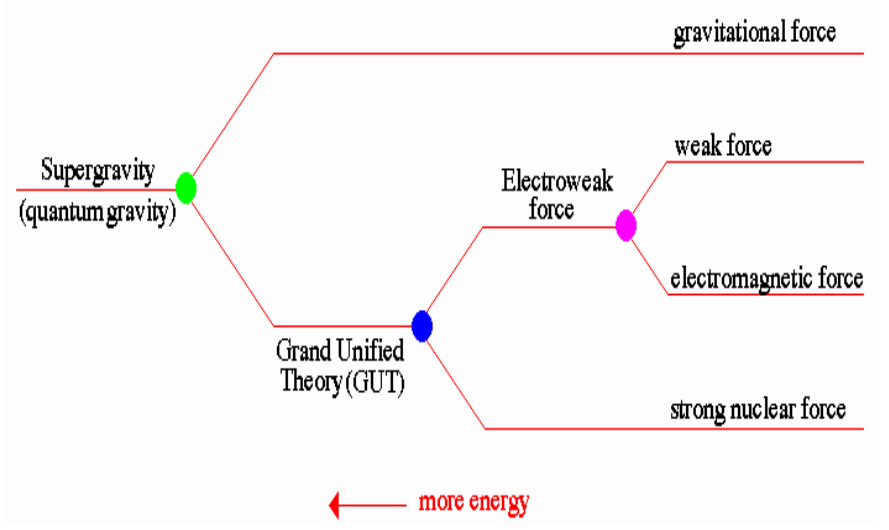

Fig 2: Unification of four forces/interactions

\section{HISTORICAL BACKGROUND AND PROGRESS IN UNIFIED FIELD THEORY}

The first unifying scientific principle was the atomic hypothesis expressed by Democritus in $400 \mathrm{BC}$. Sir Isaac Newton's theory of gravity was the first grand success to unifying in a precise mathematical framework of the laws that govern the motion of apples and planet. It incites the search for similar unifying theories that would explain ordinary matter.

The next major step in the quest for unification of electricity and magnetism was developed by Maxwell. In 1820 Hans Christian Oersted discovered that electric currents exerted forces on magnets, while in 1831, Michael Faraday made 
the observation that time-varying magnetic fields could induce electric currents. Until then, electricity and magnetism had been thought of as unrelated phenomena. In 1864, Maxwell published his famous paper on a dynamical theory of the electromagnetic field. This was the first example of a theory that was able to encompass previous separate field theories (namely electricity and magnetism) to provide a unifying theory of electromagnetism. It explained many electric and magnetic phenomena that had been discovered over the years as manifestations of a single entity, the electromagnetic field. It also had many consequences. The most important of these was the prediction of the existence of electro-magnetic wave and demonstration that light was such a wave. Thus optics was unified with electro-magnetism. Hertz in 1888 presented the decisive experimental confirmation for Maxwell theory. By 1900, the three great branches of physics, electricity, magnetism and optics had merged into a single unified theory.

Albert Einstein in 1916 successfully formulated general theory of relativity which explained gravity as consequences of dynamic of the metric field of space time and unifying structure of geometry of the space time with gravitation, dreaming of a unified theory of all the forces of the nature and of all forms of matter. In the years following the creation of the general theory, a large number of physicists and mathematicians enthusiastically participated in the attempt to unify the then-known fundamental interactions. In view of later developments in this domain, of particular interest are the theories of Hermann Weyl of 1919, who introduced the concept of an (electromagnetic) gauge field in a classical field theory and, two years later, that of Theodor Kaluza, who extended General Relativity to five dimensions. Continuing in this latter direction, Oscar Klein proposed in 1926 that the fourth spatial dimension be curled up into a small, unobserved circle. In Kaluza-Klein theory, the gravitational curvature of the extra spatial direction behaves as an additional force similar to electromagnetism. These and other models of electromagnetism and gravity were pursued by Albert Einstein in his attempts at a theory. Although it is now believed that Einstein's attempt to explain quantum mechanics in a classical field theory was unsuccessful, many believe that the goal of unification is achievable.

The development of quantum mechanics in the 1920s and throughout the twentieth century enabled the completion of the atomic program, unifying chemistry and atomic physics. All the properties of ordinary matter, in all of its variety of forms, can be explained in terms of atoms and the electromagnetic forces between them, realizing Democritus's vision. Quantum mechanics also provides for a theory of the structure of atoms in terms of the electromagnetic forces between the atomic nucleus and the electrons orbiting them.

In 1963 Sheldon Glashow proposed that the weak nuclear force and electricity and magnetism could arise from a partially unified electroweak theory. In 1967, Abdus Salam and Steven Weinberg independently revised Glashow's theory by having the masses for the $\mathrm{W}$ particle and $\mathrm{Z}$ particle arise through spontaneous symmetry breaking with the Higgs mechanism. This unified theory was governed by the exchange of four particles: the photon for electromagnetic interactions, a neutral $\mathrm{Z}$ particle and two charged $\mathrm{W}$ particles for weak interaction. As a result of the spontaneous symmetry breaking, the weak force becomes short range and the $\mathrm{Z}$ and $\mathrm{W}$ bosons acquire masses of 80.4 and $91.2 \mathrm{GeV} / \mathrm{c}^{2}$, respectively. Their theory was first given experimental support by the discovery of weak neutral currents in 1973. In 1983, the $\mathrm{Z}$ and W bosons were first produced at CERN by Carlo Rubbia's team. For their insights, Salam, Glashow and Weinberg were awarded the Nobel Prize in Physics in 1979. Carlo Rubbia and Simon van der Meer received the Prize in 1984. In 1974, Sheldon Glashow and Howard Georgi proposed unifying the strong and electroweak interactions into GeorgiGlashow model, the first Grand Unified Theory, which would have observable effects for energies much above $100 \mathrm{GeV}$.

In the latter half of the twentieth century, Physicists successfully unified the strong nuclear interaction and weak nuclear interaction with quantum 
electrodynamics to form the standard model of physics. This quantum theory of field identified the basic constituents of matter. They are the quarks that make up the nuclei at the centre of atoms and the leptons (such as the electron) that revolve about the nuclei. The standard Model also explains the forces that act on the elementary particles (the electromagnetic, the weak, and the strong or nuclear forces) as consequences of local gauge symmetries.

Beyond the standard model, theories called 'Grand Unified Theories' have been proposed to unify the electroweak force with the strong force. The theory of super symmetry requires a whole new set of particles beyond the standard model complements; a heavy partner of each quark, lepton and gauge bosons of old set together all of them making up one great superfamily of particles. The three forces strong, electromagnetic and weak all have exactly equal strength in this theory at a very high energy. Meanwhile theoretical studies range far and wide in a search for the theory of everything (TOE). The most familiar is string theory which pictures particles as infinitesimal little vibrating loop of strings in 10 dimensions. Further refinement lead to Membrane Theory, with the entire universe regarded as existing on multidimensional sheets or membranes, with particles as loops anchored on our sheet and graviton ranging into the continuum between sheets

\section{CONCLUSION}

Unified field theory, an attempt to unify the fundamental forces between the elementary particles into a single theoretical framework, is highly theoretical, and to date there is no absolute evidence that it is possible to unify gravity with the other forces. History has shown that other forces could be combined, and many physicists are willing to devote their lives, careers, and reputations to the attempt to show that gravity, too, can be expressed quantum mechanically. The consequences of such a discovery, of course, cannot be fully known until a viable theory is proven by experimental evidence. The current quest for a unified field theory is largely focused on the theory which has to be successfully included in a theory of everything. Although electromagnetism the strong and weak nuclear forces have long been explained by a single theory known as the Standard Model; gravitation does not fit into the equation. Some theoretical physicists currently believe that a quantum theory of general relativity may require frameworks other than field theory itself, such as string theory or gravity. Physicist are finding that certain depth and abstract mathmatical symmetries underling the world of subatomic particles and the force that act on them. Experiences teaches that each stage of unification leaves many questions unanswered and reveals new mysteries that only find their explanation at the next stage of unification

\section{REFERENCES}

[1] Alan Isaacs, 1996, Dictionary of Physics, Oxford University Press New York

[2] Arthur Beiser, 1999, Concept of Modern Physics, Fifth Edition, Mc GRAW Hill New York

[3] David J. Griffith, 1989, Introduction to Electrody-namics, second Edition : Prentice Hall of India Private Limited, New Delhi

[4] Encyclopedia Britanica, 2010

[5] John S. Rigden, 2000, Building Blocks of matter, McGrow hill, Newyork.

[6] Microsoft corporation, Encarta, 2009, Microsoft corporation

[7] Nari Mistry, 2000, A brief introduction to particle physics, Cornell University,USA

[8] Niel Walet, 2003, Nuclear and Particle Physics, UMIST, Manchester UK

[9] Poudel P., Gautam A., Adhikari B., Thapa M., Khatry M., 2010, Principles of Physics for Class XI and XII,Ayam Publication Kathmandu, Nepal

[10] Roger J Blin Stoyle, 1997, EUREKA ! Physics of particles, matter and the universe:Institute of physics publishing, Bristol Philadelphia, USA

[11] Wikipedia, the free encyclopedia, , en. wikipedia. org/wiki(obtained:2013.07)

[12] Young and Freedman, 2009, University Physics, $12^{\text {th }}$ Edition, Pearson Education, New Delhi, India 